\title{
Association between metabolic risk, oxidative stress and rotating shift work in a tertiary health care facility
}

\author{
Ravish H. Gowda ${ }^{\mathrm{a}}$, Gautham Melur Sukumar ${ }^{\mathrm{b}}$, Srinivas H. Gowda, ${ }^{\mathrm{c} *}$ \\ ${ }^{a}$ Department of Neurochemistry, National Institute of Mental Health and Neurosciences, Bangalore, India \\ ${ }^{\mathrm{b}}$ Department of Epidemiology, National Institute of Mental Health and Neurosciences, Bangalore, India \\ ${ }^{\mathrm{c}}$ Department of Biochemistry, Maulana Azad Medical College, New Delhi, India
}

\section{A R T I C L E I N F O}

\section{Keywords:}

Night shift work

Metabolic risk

Health care personnel

Oxidative stress

\begin{abstract}
A B S T R A C T
Context: Healthcare industry employees, work in rotating shift duties to provide $24 / 7$ services. Shift work is known to disrupt circadian rhythm and predispose the employees to metabolic risk and oxidative stress, subsequently increasing the risk for Non-Communicable Diseases. Present study attempts to evaluate this association.

Aims: To evaluate the association between metabolic risk and oxidative stress among health care personnel working in rotating night-shift as compared to dayshift.

Settings and design: Cross sectional study.

Methods and materials: Eligible 124 employees [(night shift (61), Day shift (63)] working in a tertiary care hospital in Bengaluru were randomly selected and tested for anthropometric and laboratory parameters pertaining to metabolic risk and oxidative stress and categorized accordingly using standard criteria. Statistical tests (student $t$-test) were applied to test for association between shift work, metabolic risk and oxidative stress.

Results: Metabolic risk and Oxidative stress were significantly higher in rotating night shift workers as compared to day shift $(\mathrm{p}<0.001)$ and $(\mathrm{p}<0.017)$ respectively.

Conclusion: Rotating night shift work is associated with increase in metabolic risk and oxidative stress and there is a need to consider this enhanced risk during periodical medical examination and employee health interventions. There is a need for further research to quantify risk for metabolic risk and understand this association in other occupations as well.
\end{abstract}

\section{Introduction}

Increase in modernization has increased the proportion of workforce in India along with the rise in Non-Communicable Diseases (NCDs) leading increase in Disability-Adjusted Life Year (DALY). DALY quantifies the burden of disease from mortality and morbidity, which means one DALY can be thought of as one lost year of "healthy" life loss which is about $62 \%$ in India. ${ }^{1}$.The workplace setting has contributed to increase burden of $\mathrm{NCDs}^{2}$ and there is need for undertaking workplace interventions to reduce NCDs. ${ }^{3}$ Rotating night shift work as a predisposing factor for NCD risk is often debated in peer circles. Regular exposure to long working nights and frequent disruption of sleep are linked to metabolic risk. ${ }^{4}$ Circadian rhythm of hypothalamus is important for maintaining sleep/wake cycle, feeding behaviour, central and peripheral tissue metabolism, disruption of which has major effect on energy metabolism. ${ }^{5-9}$ Rotating night shift, changes the melatonin levels thereby altering antioxidant enzyme activity and mRNA levels of such antioxidant enzymes, thereby increasing oxidative stress. ${ }^{10}$ Epidemiological studies have suggested that a Circadian rhythm alteration leads to metabolic syndrome (MS) which increases the risk for stroke and Myocardial infarction. ${ }^{5,11-13}$ Healthcare industry stands second in the number of workers reporting short sleep duration thus disrupting the circadian rhythm. ${ }^{2}$ This present study seeks to evaluate the rotating night shift healthcare workers for Metabolic, Oxidative and Anthropometric alterations.

\section{Subjects and methods}

It is a cross-sectional study conducted among health staff (aged between 20 and 60 years) working in day shift and rotating night shift,

\footnotetext{
* Corresponding author.

E-mail addresses: docravish@nimhans.ac.in, neuroravish@gmail.com (R.H. Gowda), drgauthamnimhans@gmail.com, drgauthamms@nimhans.ac.in (G.M. Sukumar), messagesrinih@gmail.com, bgowda777@gmail.com (S.H. Gowda).
} 
in a tertiary health care institution in Bengaluru, between January-August 2016. Employees who reported working at least 5 night duties (8p.m.-8 a.m. expected $12 \mathrm{hrs}$ duty/day) per month, for the past two years, were considered as employed in rotating night shift work. Employees working between 9:00 a.m. and 4.30 p.m. (expected to work for $7.5 \mathrm{hrs}$ duty/day) were considered as day shift workers. ${ }^{14}$

Workers reporting tobacco/alcohol use, self-reported inadequate physical activity and those with less than 2 years service duration were excluded from the study. Minimum of 2 years of service duration was considered as exclusion criteria as per literature review by Lajoie $\mathrm{P}$ et al. (2015), ${ }^{14}$ which indicated that minimum exposure duration of two years is necessary for development of metabolic risk. Individuals on Tobacco, alcohol users and those with physical inactivity were excluded as they are commonly associated with oxidative stress and metabolic risks.

\subsection{Method of collection of data}

Permission and support was sought from Institution authorities. List of employees from clinical and non-clinical departments employed in the institution served as the sampling frame. Subjects were selected by simple random sampling and a questionnaire was administered to gather information on age, service duration, tobacco, alcohol use and physical activity. Employees reporting tobacco, alcohol use and inadequate physical activity and not willing to give informed consent were excluded. A convenient sample size of 60 was considered, based on review of literature as most similar studies were conducted on sample size between $40-60 .{ }^{15-17}$ The process was repeated until a minimum sample size of 60 was obtained in both the groups. Finally, we recruited and studied 124 employees of which 63 were Day shift employees (non-clinical departments in medical college) and 61 were rotating night shift employees (clinical department employees).

Each of the eligible study subjects was contacted in person by the investigator and a pre-tested semi-structured questionnaire was administered to gather information on age, designation, shift work, duties per week and service duration. Anthropometric measurements (Waist Circumference, Height in $\mathrm{cm}$ and weight in $\mathrm{kg}$ ) were taken. Waist circumference was measured at the highest point of the iliac crest. Reading was taken in standing position and at the end of expiration according to standard convention. Venous blood sample was taken for analysis of the following parameters which was conducted using standard laboratory methods:

Blood glucose levels: Measured by Glucose oxidase method using spectrophotometer.

Total cholesterol: Measured by Zak's method using spectrophotometer.

MDA: Measured by Thiobarbituric acid (TBA) method using spectrophotometer.

CRP: Measured by ELISA.

Total Thiols: Measured by Ellman's method (DTNB).

Catalase: Measured by Goth's method using spectrophotometer.

Total proteins: Measured by Biuret method using spectrophotometer.

Blood pressure was measured with two subsequent measurements and mean of two measurements was taken. Standard methods were used for analysis of blood pressure.

\subsection{Metabolic risk criteria}

Operational definitions of metabolic risk have been proposed by different institutions, according to World Health Organization and the National Cholesterol Education Program, which includes similar criteria for central obesity, hyperglycaemias, dyslipidaemia and high blood pressure. Here we have considered according to the NCEP ATP III definition. ${ }^{18}$ A participant was deemed to have the metabolic risk when three or more of the following criteria were satisfied: 1) waist circumference $102 \mathrm{~cm}$ in men and $88 \mathrm{~cm}$ in women; 2) Total cholesterol $200 \mathrm{mg} / \mathrm{dl} \mathrm{3}$ ) blood pressure $130 / 85 \mathrm{mmHg}$ or known treatment for hypertension; and 4) Blood glucose level of $140 \mathrm{mg} / \mathrm{dl}$ or known treatment for diabetes. According to these criteria metabolic risk is identified when more than or equal to 3 of the above conditions are present in the same individual.

Body Mass index (weight in $\mathrm{kg} /$ height in meters square) was computed and WHO criteria was followed, accordingly adults are considered healthy weight, if their BMI is 18.5-24.9, overweight if their BMI is 25-29.9, and obese if their BMI is 30 or above.$^{19}$

Oxidative stress criteria: Oxidative stress was evaluated with reference to Sikka SC.et al. ${ }^{20}$ Oxidative stress was defined as persons with either elevated Serum Thiols or MDA measurements (MDA $>0.01$ mmol/L and Thiols. $>0.48 \mathrm{mmol} / \mathrm{L}$ ).

\subsection{Statistical analysis}

Quantitative data following normal distribution (Blood glucose, Blood pressure, Body mass index, serum Cholesterol, Waist circumference, Thiols, MDA, serum Catalase and serum Total proteins) were presented as mean and standard deviation. Categorical data (Metabolic risk, Oxidative stress and Antioxidants status) was presented as frequency and percentages. Gender and age distribution between the two study groups were compared using chi-square test to evaluate role of selection bias. Shapiro-wilk test was applied to test for normality. Based on the outcomes of normality tests, Independent ' $t$ ' test was applied to test for significant differences in mean levels of laboratory parameters between the study subjects. Chi-square test was applied to test for association between metabolic risk, oxidative stress and rotating night shift work. Results were considered significant at $\mathrm{p}<0.05$.

Ethical clearance was obtained from the institutional ethics committee of Sapthagiri Institute of Medical Sciences and Research Centre, Bengaluru.

\section{Results}

\subsection{General characteristics of study subjects}

We studied 61 workers who were predominantly on rotating night shift and 63 workers working in day shift. There were no significant differences in gender or age distribution between the two groups. Nearly $60 \%$ of rotating night shift workers was involved in clinical nature of work which was significantly higher as compared to day-shift workers (39\%). (Table 1). Metabolic risk assessment was done using blood glucose, systolic/diastolic blood pressure, mean serum total cholesterol, BMI and waist circumference. We observed that in night shift working group, mean blood glucose (107.1 \pm 36.2$)$, systolic blood pressure $(129.6 \pm 13.2)$, mean serum total cholesterol $(210 \pm 47.1)$ and waist circumference, were significantly higher than day shift workers (Table 2). Blood glucose levels were significantly higher, $17 \mathrm{mg} / \mathrm{dl}$ higher in rotating night shift working individuals. Diastolic blood pressure, though observed to be higher in night shift workers, the difference was not significant.

In the Oxidative stress assessment, we measured serum thiols, CRP and MDA. The Mean serum thiols $(0.72 \pm 0.39)$ were higher in night shift working group compared to day shift group $(0.66 \pm 0.34)$. Similarly, Mean Serum MDA (0.0351 \pm 0.04$)$, CRP (5.09 \pm 2.8$)$ was observed to be significantly higher in night shift workers then day shift $(\mathrm{MDA}=0.01 \pm 0.00, \mathrm{CRP}=1.09 \pm 1.07)$ (Table 2$)$. However, only MDA and CRP showed statistically significant difference.

Antioxidant status was measured using serum Catalase and total protein. We observed mean Catalase levels $(1165.57 \pm 278.2)$ were significantly lower in night shift workers compared to day shift group $(1378.01 \pm 393.1)$.

In the Anthropometry assessment, the mean Waist Circumference in night shift group was $(96.81 \pm 14.13)$, significantly higher than the 
Table 1

Descriptive statistics of study subjects.

\begin{tabular}{|c|c|c|c|c|}
\hline & $\begin{array}{l}\text { Rotating night } \\
\text { shift }(\mathrm{n}=61) \\
\mathrm{N}(\%)\end{array}$ & $\begin{array}{l}\text { Day shift } \\
(\mathrm{n}=63) \\
\mathrm{N}(\%)\end{array}$ & $\begin{array}{l}\text { Total } \\
(124)\end{array}$ & $\mathrm{p}$ value \\
\hline \multicolumn{5}{|l|}{ Gender $^{\mathrm{a}}$} \\
\hline Male & $23(46.9)$ & $26(53.1)$ & 49 & 0.68 \\
\hline Females & $38(50.7)$ & 37 (49.3) & 75 & \\
\hline \multicolumn{5}{|l|}{ Nature of work $\mathrm{k}^{\mathrm{a}}$} \\
\hline Clinical & $46(60.5)$ & $30(39.5)$ & 76 & $0.001 *$ \\
\hline Non-clinical & $15(31.2)$ & $33(68.8)$ & 48 & \\
\hline \multicolumn{5}{|l|}{ Age group $^{a}$} \\
\hline$\leq 30$ years & $27(57.4)$ & $20(42.6)$ & 47 & 0.15 \\
\hline$>30$ years & $34(44.2)$ & $43(55.8)$ & 77 & \\
\hline $\begin{array}{l}\text { Age in Median years, } \\
\text { (intra quartile } \\
\text { range) })^{\mathrm{b}}\end{array}$ & 33 years (19) & 38 years (23) & $\begin{array}{l}36 \text { years } \\
(22)\end{array}$ & 0.18 \\
\hline
\end{tabular}

Age group is divided into two group less than 30 years and more than 30 years. $* \mathrm{p}<0.05$ is taken as statistical significant.

a Chi-square test is applied for gender, age group and nature of work and data presented as frequency number and percentage.

b Mann Whitney $U$ Test is applied for age and data presented as median.

day shift $(92.8 \pm 14.13)$ and night shift workers BMI $(26.7 \pm 4.1)$ were not significantly different from day shift worker BMI $(26.8 \pm 3.6)$.

Metabolic risk and oxidative stress are grouped into high and low risk. Metabolic risk is grouped according NCEP ATPIII criteria and oxidative stress is grouped according to serum thiol/MDA levels (high group when their MDA $>0.01 \mathrm{mmol} / \mathrm{L}$ and Thiols $>0.48 \mathrm{mmol} / \mathrm{L}$ ). Antioxidant status is grouped into low risk if the catalase level is more than $1100 \mathrm{ku} / 1$ with normal total protein levels. The frequency data among high risk group was compared between night shift and day shift workers using chi square and data presented as odd ratio and $\mathrm{p}$ value. The odds of metabolic risk was 2.53 times (95\% CI, 1.2to 5.2) higher among rotating night shift individuals compared to day shift workers. Similarly, the odds of high oxidative was 4.84 times (95\% CI 1.88 to 12.4) higher in night shift workers (Table 3). Female's subjects with rotating night shift work had higher metabolic risk, oxidative stress and lower antioxidants compared to male rotating night shift workers (Fig. 1).

\section{Discussion}

Any biological process in the body that repeats itself over a period of approximately $24 \mathrm{~h}$ and maintains this rhythm in the absence of external stimuli is considered a circadian rhythm. ${ }^{5,21}$ The major zeitgeber (Keeper) for our body's circadian rhythm is light and thereby regulating endocrinological signals downstream. ${ }^{6}$ The master Circadian clock is located in suprachiasmatic nuclei (SCN). Since we are in the age of modernization, many healthcare industries need to function around the clock and thus there is demand for night shift work also, this puts pressure on healthcare professionals to be awake and functional at odd hours of the night. Due to exposure to light at odd times, downstream signalling of SCN gets hampered, leading to dysregulation of endocrine signals, resulting in increase in the non-communicable disease risk (Fig. 2). ${ }^{22}$ Developing countries like India have burden of increasing non-communicable disease and fast becoming major burden in modernization society and is reducing the productivity. ${ }^{23}$ Insufficient sleep, changes in dietary habits, regularly exposed to working long nights and frequent waking up in night, which are the feature of night shift work schedules, have shown to be linked with metabolic risk. ${ }^{4}$

World health organization has observed increase in the risk of cancers due to reduced melatonin secretion caused by light exposure during nights. ${ }^{24}$ US Government included sleep health programs in its Healthy people 2020 initiative recognising the adverse effect of insufficient sleep. ${ }^{2}$ Night shift work carries considerable heath risk and our objective of the present study was to find the metabolic risk, oxidative stress and antioxidant status among health care personnel working in rotating night shift as compared to those in day shift working in South India.

\subsection{Brain glucose utilization during night shift work}

Brain is the major organ for the utilization of blood glucose during sleep, it uses $50 \%$ of blood glucose during fasting state by insulin dependent manner. Studies have shown there is reduced glucose tolerances and increase in insulin resistance leading to metabolic syndrome in night shift work, since brain is major organ utilising the glucose during resting period, insulin resistance might lead to reduces utilisation of glucose by brain and further contribute to increase in blood glucose concentration. ${ }^{8,9}$ In our study we observed blood glucose levels in night shift group was $17 \mathrm{mg} / \mathrm{dl}$ higher than day shift population. Supporting our findings there were evidences regarding association between blood glucose and shift work by Matheson A et al. ${ }^{25}$ and Padilha et al. ${ }^{26}$. Li Y et al. (2011), have showed that insulin resistance leading to metabolic syndrome was more common in night shift workers than in day workers. ${ }^{27}$ But in contrast, Biggi et al. found no consistent effect of permanent night work on fasting glucose or insulin resistance. $^{28}$

\subsection{Hypertension and night shift}

BMI and antioxidant status are known to alter blood pressure in

Table 2

Comparison of means of metabolic, oxidative stress and anti-oxidant parameters among study groups.

\begin{tabular}{|c|c|c|c|}
\hline Parameters & Rotating night shift $(n=61)$ & Day shift $(n=63)$ & $\mathrm{p}$ value \\
\hline Blood glucose (mg/dl) & $107.1 \pm 36.2$ & $89.9 \pm 25.8$ & $0.001^{*}$ \\
\hline Systolic Blood pressure (mm/Hg) & $129.6 \pm 13.2$ & $120.6 \pm 7.5$ & $0.001 *$ \\
\hline Body Mass Index & $26.7 \pm 4.1$ & $26.8 \pm 3.6$ & 0.191 \\
\hline Serum Total cholesterol $(\mathrm{mg} / \mathrm{dl})$ & $210 \pm 47.1$ & $196.5 \pm 32.3$ & $0.001 *$ \\
\hline Waist Circumference (cms) & $96.81 \pm 10.59$ & $92.8 \pm 14.13$ & $0.004 *$ \\
\hline MDA (mmol/L) & $0.04 \pm 0.04$ & $0.01 \pm 0.00$ & $<0.001 *$ \\
\hline \multicolumn{4}{|l|}{ Anti-oxidant parameters } \\
\hline Catalase (KU/L) & $1165.57 \pm 278.2$ & $1378.01 \pm 393.1$ & $0.001 *$ \\
\hline Total Proteins (gm/dl) & $9.9 \pm 2.7$ & $11.4 \pm 3.7$ & $0.019 *$ \\
\hline
\end{tabular}

Independent student $t$-test was applied to compare between group and data are represented as means $\pm \mathrm{SD}$, *p $<0.05$ is considered statically significant. CRP:C reactive protein, MDA:Malondialdehyde. 
Table 3

Association between metabolic risk, oxidative stress, antioxidant status and shift workers.

\begin{tabular}{|c|c|c|c|c|}
\hline Parameters & $\begin{array}{l}\text { Rotating night shift }(\mathrm{n}=61) \\
\mathrm{N}(\%)\end{array}$ & $\begin{array}{l}\text { Day shift }(\mathrm{n}=63) \\
\mathrm{N}(\%)\end{array}$ & Total n (\%) & $\mathrm{p}$ value (Merged) \\
\hline Metabolic Risk (high risk group) & $26(42.6 \%)$ & $2(3.2 \%)$ & $28(22.6 \%)$ & $<0.001^{*}$ \\
\hline Blood glucose & $12(19.7 \%)$ & $3(4.8 \%)$ & $15(12.09 \%)$ & \\
\hline Hypertension & $29(47.5 \%)$ & $8(12.7 \%)$ & $37(29.8 \%)$ & \\
\hline Elevated BMI & $28(45.9 \%)$ & $30(47.6 \%)$ & $58(46.77 \%)$ & \\
\hline Serum Total cholesterol (mg/dl) & $28(45.9 \%)$ & $25(39.7 \%)$ & $53(42.7 \%)$ & \\
\hline $\mathrm{CRP}(\mathrm{mg} / \mathrm{ml})$ & $42(68.9 \%)$ & $1(1.6 \%)$ & $43(34.6 \%)$ & \\
\hline Oxidative Stress parameters (high risk group) & $34(55.7 \%)$ & $32(50.8 \%)$ & $66(53.2 \%)$ & $0.017^{*}$ \\
\hline Thiols Estimation (mmol/L) & $43(70.5 \%)$ & $47(74.6 \%)$ & $90(72.5 \%)$ & \\
\hline $\mathrm{MDA}(\mathrm{mmol} / \mathrm{L})$ & $44(72.1 \%)$ & $8(12.7 \%)$ & $52(41.9 \%)$ & \\
\hline Anti-oxidant status (low risk group) & $13(21.3 \%)$ & $52(82.5 \%)$ & $65(52.4 \%)$ & $<0.001^{*}$ \\
\hline Catalase (KU/L) & $12(19.7 \%)$ & $49(80.3 \%)$ & $61(49.1 \%)$ & \\
\hline Total Proteins (gm/dl) & $1(1.6 \%)$ & $9(14.3 \%)$ & $10(8.06 \%)$ & \\
\hline
\end{tabular}

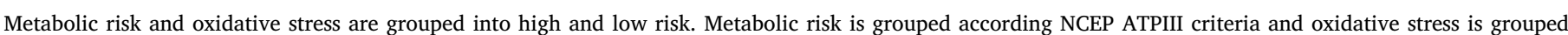

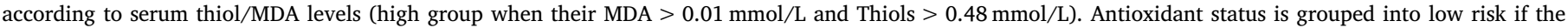

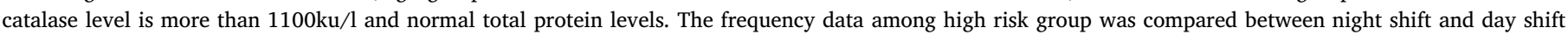
workers using chi square and p value $<0.05$ is taken as statistically significant.

essential hypertension patients. ${ }^{29,30}$ Normally blood pressure decreases by $10-20 \%$ during sleep compared to daytime. This is mediated by decrease in sympathetic output during nocturnal sleep. Sleep disturbances leads to increase in sympathetic nervous activity thereby increase systolic blood pressure. ${ }^{31}$ We observed in our study, their was increase in systolic blood pressure around $9 \mathrm{mmHg}$ higher in rotating night shift individuals and systolic blood pressure showed more difference than diastolic blood pressure. Puttonen S et al. (2009) in their studies have shown a higher risk of hypertension in men than women in the rotating shift working group, In their study on 712 men and 831 women, they have shown the men had higher Blood pressure even after adjusting to all confounding factors such as adjustment for age and risk factors, such as low socio-economic position, job strain, smoking, diet, family history of CHD, physical inactivity, alcohol consumption, obesity, homocysteine, C-reactive protein and lipids. The mechanism they have observed is the increase cardiac artery intima media and in women they did not find any association between shift work and carotid atherosclerosis indicators. ${ }^{32}$ The prevalence of hypertension according to 2016 update in India was $22.6 \%$ of male population in the age group of 35-44 years whereas $18.3 \%$ of females. ${ }^{33}$ But in our study, we observed women where at higher risk compared to men in the rotating night work group (29\% women had higher risk compared $22 \%$ men). However, there were limitations in the present study, study did not account for diet, noise among work place, leisure time given etc. Thus, future studies to confirm the association between hypertension and gender are necessary to account for other confounding factors.

\subsection{Inflammatory marker and night shift}

Many studies have shown that inflammatory marker CRP raise with reduce sleep duration. ${ }^{34-37} \mathrm{CRP}$ is correlated with systemic inflammation and studies shows that IL- 6 and CRP levels during night shift are inversely correlates with melatonin. ${ }^{37,38}$ CRP elevation even moderate for longer duration is always considered as presence of chronic inflammation. ${ }^{37}$ In our study, there was a significant increase in CRP levels $4.021 \mathrm{mg} / \mathrm{dl}$ higher in night shift group compared day shift group. So increased CRP due to reduced sleep could lead to systemic inflammation, but CRP is a nonspecific inflammatory marker and there are factors that might have role in association between night work and low grade inflammation including circadian disruption, poor sleep quality and reduce sleep duration. Nevertheless CRP can be a useful biomarker to assess the risk of future diabetes and metabolic syndrome, ${ }^{39}$ it could be used to monitor metabolic risk in night shift workers.

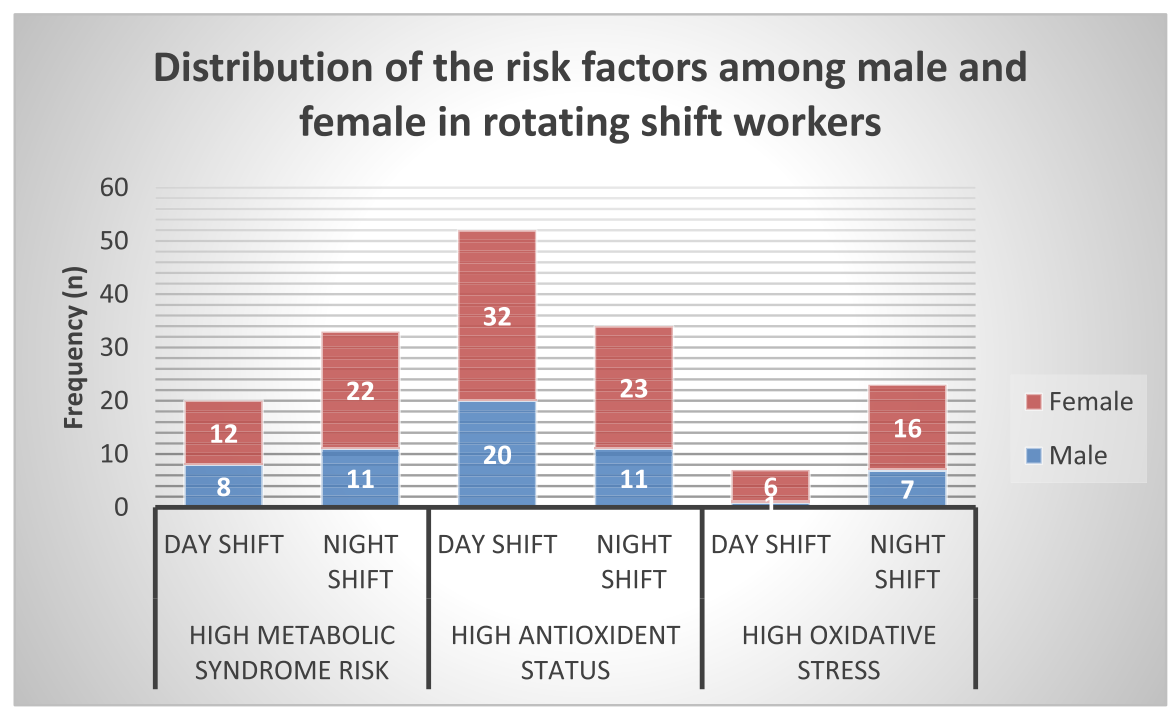

Fig. 1. Gender specific distribution of Metabolic risk, oxidative stress and antioxidant status among night shift and day shift groups. Frequency of individual effected by high metabolic risk, high oxidative stress and high antioxidant status among male and female distributed between night and day shift workers. 


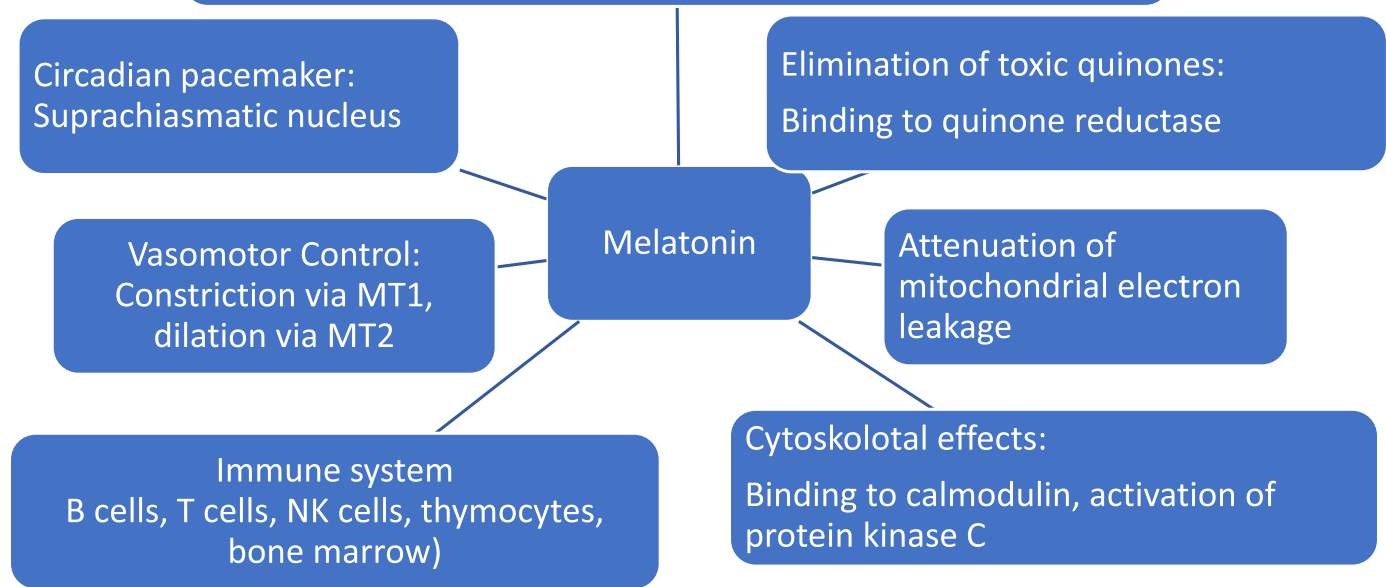

Fig. 2. Schematic representation of Melatonin related pathways.

Shows the various pathway in which metabolic and oxidative stress are linked with melatonin Pandi-Perumal SR et al., 2016) ${ }^{62}$.

\subsection{Adipocyte dysregulation and night shift}

Many studies have been documented regarding role of weight gain, dietary choices and physical activity levels in causing metabolic risk. ${ }^{40}$ In addition to these know factors which leads to metabolic risk, studies on Japanese population by Obayashi K et al., have observed that elderly people exposed to illuminated rooms lead to higher body weight, BMI and Waist circumference. ${ }^{41}$ Fonken LK et al. (2013) in their study observed that exposure of light for increased duration during night leads to compromised metabolic processes disrupting the normal circadian rhythms and thereby increases the risk of obesity. ${ }^{40}$ Disruption of circadian rhythms leading to obesity was also demonstrated in animal studies by Froy $\mathrm{O}$ et al., they reported mammals develops obesity features due of light and dark cycles. ${ }^{42}$ Molecular basis of the development of obesity due to disruption of circadian rhythm is described by Yang $\mathrm{X}$ et al. (2006), they have studied the circadian genes related to nuclear receptors such as Rev-erb, PPAR and ROR subfamilies which are affected by the disruption of light dark cycle and directly affect metabolism. ${ }^{43}$ Phenotypic expression of obesity has multifaceted mechanism involving various mutations in clock genes, variations in metabolism, drop in thermogenic response, disruption of neurohumoral parameters like leptin and ghrelin and impairment in genes present in adipocyte. ${ }^{44}$ The disruption of the light dark cycle also plays an important role in developing obesity phenotype, since Sun is our important source of the light, the human body is evolved around the light dark cycle centred around the sun light. Sun provides bright light during the day and virtually no light at night and our endogenous circadian rhythms such as core body temperatures, cardiovascular activity, endocrine system, renal activity, gastrointestinal tract motility, many body metabolism, gene expression and hormone productions have an oscillatory type synced to this day night cycle. Rhythms are reset every day in $24 \mathrm{~h}$ cycle with the exposure of sun light and a type of physiological anticipation for many body metabolisms exist. Electric light, by contrast, is dim and ill-timed, disrupting all aspects of our endogenous circadian rhythmicity. The intensity and spectral content of electric light are often not adequate for proper circadian resetting and are too much during the night for a true 'dark' to be detected. This can lead to 'circadian disruption' compromising general well-being and perhaps increasing risk of a variety of specific diseases. ${ }^{45}$ In humans, increase in obesity is caused by Engineered Junk Foods, excessive food addiction, Insulin resistance, certain medications, Leptin Resistance, genetic predisposition and hormonal imbalances, but it is also contributed by our sleeping patterns and eating habits at late night. ${ }^{46}$ Sleeping patterns alters the circadian rhythm and disruption normal metabolic processing leading to development of insulin resistances and obesity. Eating excessive and during late night also has adverse effect on the body metabolism, the energy requirement is at minimum during the night time and eating during night will lead to deposition of the excess calorie in adipose tissue contributing to obesity. Epidemiological studies done by Knutson KL et al., have demonstrated association of reduced sleep duration with increased body mass index and diabetes. ${ }^{46}$ Further, sympathetic nervous system activity is elevated due to working late night, this in turn leads to increase in free fatty acids which further contribute insulin resistance ${ }^{47}$

Similar to these studies we found, waist circumference higher in night shift workers compared to day shift workers in our study population, but BMI was not significantly different between groups. The difference observed might be influenced by confounding factors such as calorie intake and physical activities ${ }^{47}$ which need to be further evaluated.

\subsection{Oxidative stress and night shift}

In our study night shift workers had 4.84 times (1.88-12.4; 95\%CI) $(\mathrm{p}=0.001)$ higher oxidative stress compared to day shift workers. Studies by Akbar S et al., have shown an increase in oxidative stress during night shift. ${ }^{48}$ Experimental studies have also shown that circadian rhythm effects expression and activity of oxidative and antioxidative enzymes. ${ }^{10}$ However Ulas et al., have shown that there was no significant difference of oxidative stress parameters among day and night shift working subjects. ${ }^{17}$ Sharma A et al., demonstrated that increased marker of DNA oxidation and increased levels of urinary 8-OHDG was seen among night shift workers and also showed a positive correlation between urinary stress markers among night workers with the age group of $35-45 y r s .{ }^{16}$ Serum malondialdehyde is one such markers formed by cellular membrane lipid peroxidation due to oxidative stress and its levels are increased in neurodegenerative diseases, cardiovascular diseases and cancers. ${ }^{49-52}$ In our study we have observed significant higher MDA levels among night shift group even though levels of Total thiols were higher in night shift but not significantly different between groups. Oxidative stress leads to cytokine production, various different mechanism could increase cytokine 
production along with oxidative stress like Age, obesity, blood glucose levels, blood pressure and other metabolic risk factors make individuals more vulnerable to harmful effects of oxidative stress during night shift work. ${ }^{37,46,53}$

\subsection{Antioxidants and night shift}

In our study we have found reduce antioxidant levels among night shift worker compared to day shift workers and odds ratio for Antioxidant status of night shift workers was 3.75 times (1.6-8.5; 95\%CI) ( $\mathrm{p}=0.0016)$ lesser antioxidants compared to day shift workers. Melatonin have been found to produce antioxidants and reduce reactive oxygen species. ${ }^{54}$ Melatonin have found to alter antioxidants m-RNA expression (Catalase, SOD, Glutathione peroxidase), in mouse liver. ${ }^{55}$ Hirayama $\mathrm{J}$ et al., have shown genes like BMAL1/ CLOCK, RORs and Rev-ErbS are under direct regulation by the circadian clock transcriptional factors. ${ }^{56}$ Even in plants antioxidant TRX system is been shown to be under circadian rhythm as shown by Lemaire SD et al. ${ }^{57}$. Exposure to light reduces the melatonin synthesis, decreasing antioxidants like glutathione peroxidase, catalase and other enzymes of antioxidative pathway. Pro-oxidant levels also reduces the antioxidant levels in the boby, they tend to increases redox sensitive transcription factors such as signal transducer and activator of transcription (STAT), $\mathrm{CREB}, \mathrm{NF}-\mathrm{kB}, \mathrm{AP}-1$. Induction of these transcription factors induces inflammatory cytokines (TNFalpha, IL-1, IL-6, IL-8) and growth factors, these are shown to down regulated antioxidants through two mechanisms, 1. Direct Transcriptional factors regulation 2. By influencing PGE2 synthesis. ${ }^{58-60}$ Increase in healthcare demands has lead to difficulty in adaptation even for the trained recruits in case of night shift work. Studies says that different age group have varied adverse health outcome as shown by Ramin et al. ${ }^{15}$.

\section{Conclusions}

Our study data suggest that chronic, insufficient sleep in rotating health care workers decreases blood glucose tolerance, increased hypertension, obesity, leads to oxidative stress with decrease antioxidants, which further may contribute to increase cancer and other degenerative diseases along with metabolic syndrome.

Shift and night work appear to have a negative effect on worker health, possibly due to its impact on sleep-wake cycles, eating and exercise habits, thermogenesis, hormone secretion, and blood pressure levels. Thus, indicating that lifestyle interventions for night shift should be recommended. We should find ways to minimize the health burden of shift work. Main corrective steps to be taken is by making sleep, a priority in the healthcare professionals personal life by education, training programs to all workers as described in many health care initiative. ${ }^{61}$ Further to take preventive steps by education and training programs for all the hospital managers and healthcare professionals working during night shifts which could also give encouragements and appreciation about the services provided, thereby reducing the psychological burden of long working hours related to sleep and fatigue issues. Hospital Managers should foster the need for improving the work schedule design and promptly implement policies and systems that decrease the risk of fatigue and health problems. Healthcare workers should be periodically examined to evaluate the influence of work schedules on factors like alertness, errors in handling responsibilities and illnesses. Enough time for sleep and encouragements for good practices and behaviours to improve sleep and alertness should be encouraged. Healthcare professionals having sleeping problems should be facilitated to seek special help and support from sleep clinics. Among the life style modification, ways to avoid weight gain based on lowcalorie diets, personalized health care and nutritional orientations, in addition to physical exercise programs which can be adapted to the worker's routine. Healthcare workers need to be subjected to (1) Training programs to handle night shift work schedule. (2) To monitor their health status by regular checkup with relevant blood investigations. (3) Assurance of changing work schedules, to minimize the reduce the outcome of the night work on health. Recognizing the importance of adverse impact of prolonged night shift on health itself will go a long way in reducing its impact on health burden.

\section{Limitations}

Limitation of our study is the samples size we have included convenience sampling method and due to very stringent selection criteria, allowing the inclusion of less number of subjects, due to which we can't generalize the results completely and also study had more of young and healthy population. Population was healthcare workers, and hence, the findings cannot be generalized to other night shift workers in different occupation. Lastly the present study, was conducted in a single tertiary care teaching hospital, hence results may not be generalized. Further studies are recommended to explore more on the effects of night shift working.

\section{Conflicts of interest}

There are no conflicts of interest.

\section{Financial support}

This research did not receive any specific grant from funding agencies in the public, commercial, or not-for-profit sectors.

\section{Acknowledgement}

We are grateful to Sapthagiri Institute of Medical Sciences and Research Centre, Bangalore for providing ethical clearance and infrastructure.

\section{References}

1. Puttonen S, Härmä M, Hublin C. Shift work and cardiovascular disease - pathways from circadian stress to morbidity. Scand J Work Environ Health. 2010 Mar;36(2):96-108.

2. Luckhaupt SE, Tak S, Calvert GM. The prevalence of short sleep duration by industry and occupation in the National Health Interview Survey. Sleep. 2010 Feb;33(2):149-159.

3. Sukumar GM, Kupatira K, Gururaj G. Feasibility of integrating mental health and noncommunicable disease risk factor screening in periodical medical examination of employees in industries: an exploratory initiative. Indian J Occup Environ Med. 2015;19(1):19-24.

4. Haus EL, Smolensky MH. Shift work and cancer risk: potential mechanistic roles of circadian disruption, light at night, and sleep deprivation. Sleep Med Rev. 2013 Aug;17(4):273-284.

5. Foster RG, Kreitzman L. The rhythms of life: what your body clock means to you!. Exp Physiol. 2014 Apr;99(4):599-606.

6. Husse J, Eichele G, Oster H. Synchronization of the mammalian circadian timing system: light can control peripheral clocks independently of the SCN clock: alternate routes of entrainment optimize the alignment of the body's circadian clock network with external time. BioEssays News Rev Mol Cell Dev Biol. 2015 Oct;37(10):1119-1128.

7. Kopelman PG. Obesity as a medical problem. Nature. $2000 \mathrm{Apr}$ 6;404(6778):635-643.

8. Molzof HE, Wirth MD, Burch JB, et al. The impact of meal timing on cardiometabolic syndrome indicators in shift workers. Chronobiol Int. 2017;34(3):337-348.

9. Silva-Costa A, Rotenberg L, Coeli CM, Nobre AA, Griep RH. Night work is associated with glycemic levels and anthropometric alterations preceding diabetes: baseline results from ELSA-Brasil. Chronobiol Int. 2016;33(1):64-72.

10. Rodriguez C, Mayo JC, Sainz RM, et al. Regulation of antioxidant enzymes: a significant role for melatonin. J Pineal Res. 2004 Jan;36(1):1-9.

11. Alibhai FJ, Tsimakouridze EV, Reitz CJ, Pyle WG, Martino TA. Consequences of circadian and sleep disturbances for the cardiovascular system. Can J Cardiol. 2015 Jul;31(7):860-872.

12. Tchernof A, Després J-P. Pathophysiology of human visceral obesity: an update. Physiol Rev. 2013 Jan;93(1):359-404.

13. Gan Y, Yang C, Tong X, et al. Shift work and diabetes mellitus: a meta-analysis of observational studies. Occup Environ Med. 2015 Jan;72(1):72-78.

14. Lajoie P, Aronson KJ, Day A, Tranmer J. A cross-sectional study of shift work, sleep quality and cardiometabolic risk in female hospital employees. BMJ Open. 2015 Mar 10;5(3):e007327. 
15. Ramin C, Devore EE, Wang W, Pierre-Paul J, Wegrzyn LR, Schernhammer ES. Night shift work at specific age ranges and chronic disease risk factors. Occup Environ Med. 2015 Feb;72(2):100-107.

16. Sharma A, Tiwari S, Singaravel M. Circadian rhythm disruption: health consequences. Biol Rhythm Res. 2016 Mar 3;47(2):191-213.

17. Ulas T, Buyukhatipoglu H, Kirhan I, et al. Evaluation of oxidative stress parameters and metabolic activities of nurses working day and night shifts. Rev Esc Enferm U P. 2013 Apr;47(2):471-476.

18. Lorenzo C, Williams K, Hunt KJ, Haffner SM. The national cholesterol education program - adult treatment panel III, international diabetes federation, and World health organization definitions of the metabolic syndrome as predictors of incident cardiovascular disease and diabetes. Diabetes Care. 2007 Jan;30(1):8-13.

19. Anuurad E, Shiwaku K, Nogi A, et al. The new BMI criteria for asians by the regional office for the western pacific region of WHO are suitable for screening of overweight to prevent metabolic syndrome in elder Japanese workers. J Occup Health. 2003 Nov;45(6):335-343

20. Sikka SC. Relative impact of oxidative stress on male reproductive function. Curr Med Chem. 2001 Jun;8(7):851-862.

21. Helm B, Ben-Shlomo R, Sheriff MJ, et al. Annual rhythms that underlie phenology: biological time-keeping meets environmental change. [Internet]. Proc $R$ Soc B Bio Sci. 2013 Aug 22. [cited 2017 Sep 2];280(1765). Available from:. http://www.ncbi. nlm.nih.gov/pmc/articles/PMC3712433/.

22. Tsang $\mathrm{AH}$, Barclay JL, Oster $\mathrm{H}$. Interactions between endocrine and circadian systems. J Mol Endocrinol. 2014 Feb 1;52(1):R1-R16.

23. Boutayeb A, Boutayeb S. The burden of non communicable diseases in developing countries. Int $J$ Equity Health. 2005 Jan 14;4:2.

24. Giudice A, Crispo A, Grimaldi M, et al. The effect of light exposure at night (LAN) on carcinogenesis via decreased nocturnal melatonin synthesis. Mol Basel Swit\% 2018 May 29;23(6).

25. Matheson A, O'Brien L, Reid J-A. The impact of shiftwork on health: a literature review. J Clin Nurs. 2014 Dec;23(23-24):3309-3320.

26. Padilha HG, Crispim CA, Zimberg IZ, et al. A link between sleep loss, glucose metabolism and adipokines. Braz J Med Biol Res Rev Bras Pesqui Medicas E Biol. 2011 Oct:44(10):992-999.

27. Li Y, Sato Y, Yamaguchi N. Shift work and the risk of metabolic syndrome: a nested case-control study. Int J Occup Environ Health. 2011 Jun;17(2):154-160.

28. Biggi N, Consonni D, Galluzzo V, Sogliani M, Costa G. Metabolic syndrome in permanent night workers. Chronobiol Int. 2008 Apr;25(2):443-454.

29. Rüger M, Scheer FAJL. Effects of circadian disruption on the cardiometabolic system. Rev Endocr Metab Disord. 2009 Dec;10(4):245-260.

30. Chrysohoou C, Panagiotakos DB, Pitsavos C, et al. The implication of obesity on total antioxidant capacity in apparently healthy men and women: the ATTICA study. Nutr Metabol Cardiovasc Dis. 2007 Oct 1;17(8):590-597.

31. Bonham MP, Bonnell EK, Huggins CE. Energy intake of shift workers compared to fixed day workers: a systematic review and meta-analysis. Chronobiol Int 2016;33(8):1086-1100.

32. Puttonen S, Kivimäki M, Elovainio M, et al. Shift work in young adults and carotid artery intima-media thickness: the Cardiovascular Risk in Young Finns study. Atherosclerosis. 2009 Aug;205(2):608-613.

33. Manohar S, Thongprayoon C, Cheungpasitporn W, Mao MA, Herrmann SM. Associations of rotational shift work and night shift status with hypertension: a systematic review and meta-analysis. J Hypertens. 2017 Oct;35(10):1929-1937.

34. Ferrie JE, Kivimäki M, Akbaraly TN, et al. Associations between change in sleep duration and inflammation: findings on C-reactive protein and interleukin 6 in the whitehall II study. Am J Epidemiol. 2013 Sep 15;178(6):956-961.

35. Meier-Ewert HK, Ridker PM, Rifai N, et al. Effect of sleep loss on C-Reactive protein, an inflammatory marker of cardiovascular risk. J Am Coll Cardiol. 2004 Feb 18;43(4):678-683.

36. Patel SR, Zhu X, Storfer-Isser A, et al. Sleep duration and biomarkers of inflammation. Sleep. 2009 Feb 1;32(2):200-204

37. Wieser V, Moschen AR, Tilg H. Inflammation, cytokines and insulin resistance: a clinical perspective. Arch Immunol Ther Exp (Warsz). 2013 Apr;61(2):119-125.

38. Strohmaier S, Devore EE, Zhang Y, Schernhammer ES. A review of data of findings on night shift work and the development of DM and CVD events: a synthesis of the proposed molecular mechanisms. Curr Diab Rep. 2018 Oct 20;18(12):132

39. Demir I, Toker A, Zengin S, Laloglu E, Aksoy H. Oxidative stress and insulin resistance in policemen working shifts. Int Arch Occup Environ Health. 2016 Apr 1;89(3):407-412

40. Fonken LK, Workman JL, Walton JC, et al. Light at night increases body mass by shifting the time of food intake. Proc Natl Acad Sci U S A. 2010 Oct 26;107(43):18664-18669.

41. Obayashi K, Saeki K, Kurumatani N. Ambient light exposure and changes in obesity parameters: a longitudinal study of the HEIJO-KYO cohort. J Clin Endocrinol Metab. 2016 Sep 1;101(9):3539-3547.

42. Froy O. Circadian rhythms and obesity in mammals. [Internet]. ISRN obes. 2012 Nov 18. [cited 2018 Dec 30]; 2012.Available from:. https://www.ncbi.nlm.nih.gov/pmc/ articles/PMC3914271.

43. Yang X, Downes M, Yu RT, et al. Nuclear receptor expression links the circadian clock to metabolism. Cell. 2006 Aug 25;126(4):801-810.

44. Antunes LC, Levandovski R, Dantas G, Caumo W, Hidalgo MP. Obesity and shift work: chronobiological aspects. Nutr Res Rev. 2010 Jun;23(1):155-168.

45. Stevens RG, Zhu Y. Electric light, particularly at night, disrupts human circadian rhythmicity: is that a problem? [Internet]. Philos Trans R Soc B Biol Sci. 2015 May 5. [cited 2018 Dec 30];370(1667). Available from:. https://www.ncbi.nlm.nih.gov/ pmc/articles/PMC4375361/.

46. Knutson KL. Does inadequate sleep play a role in vulnerability to obesity? Am J Hum Biol Off J Hum Biol Counc. 2012 Jun;24(3):361-371.

47. McFadden E, Jones ME, Schoemaker MJ, Ashworth A, Swerdlow AJ. The relationship between obesity and exposure to light at night: cross-sectional analyses of over 100,000 women in the breakthrough generations study. Am J Epidemiol. 2014 Aug $1 ; 180(3): 245-250$

48. Sharifian A, Farahani S, Pasalar P, Gharavi M, Aminian O. Shift work as an oxidative stressor. J Circadian Rhythms. 2005 Dec 28;3:15.

49. Savini I, Catani MV, Evangelista D, Gasperi V, Avigliano L. Obesity-associated oxidative stress: strategies finalized to improve redox state. Int J Mol Sci. 2013 May 21;14(5):10497-10538

50. Naduthota RM, Bharath RD, Jhunjhunwala K, et al. Imaging biomarker correlates with oxidative stress in Parkinson's disease. Neurol India 2017 Mar 1;65(2):263.

51. Ho E, Karimi Galougahi K, Liu C-C, Bhindi R, Figtree GA. Biological markers of oxidative stress: applications to cardiovascular research and practice. Redox Biol. 2013 Oct 8:1:483-491.

52. Asher G, Schibler U. Crosstalk between components of circadian and metabolic cycles in mammals. Cell Metabol. 2011 Feb 2;13(2):125-137.

53. Kondratov RV. A role of the circadian system and circadian proteins in aging. Ageing Res Rev. 2007 May;6(1):12-27.

54. Zhang H-M, Zhang Y. Melatonin: a well-documented antioxidant with conditional pro-oxidant actions. J Pineal Res. 2014 Sep;57(2):131-146.

55. Xu Y-Q, Zhang D, Jin T, et al. Diurnal variation of hepatic antioxidant gene expression in mice. PLoS One. 2012 Aug 29;7(8):e44237.

56. Hirayama J, Sahar S, Grimaldi B, et al. CLOCK-mediated acetylation of BMAL1 controls circadian function. Nature. 2007 Dec 13;450(7172):1086-1090.

57. Lemaire SD, Collin V, Keryer E, Issakidis-Bourguet E, Lavergne D, Miginiac-Maslow M. Chlamydomonas reinhardtii: a model organism for the study of the thioredoxin family. Plant Physiol Biochem. 2003 Jun 1;41(6):513-521.

58. Haddad JJ. Antioxidant and prooxidant mechanisms in the regulation of redox(y)sensitive transcription factors. Cell Signal. 2002 Nov;14(11):879-897.

59. Hajjar DP, Gotto AM. Biological relevance of inflammation and oxidative stress in the pathogenesis of arterial diseases. Am J Pathol. 2013 May;182(5):1474-1481.

60. Mathy-Hartert M, Deby-Dupont GP, Reginster J-YL, Ayache N, Pujol J-P, Henrotin YE. Regulation by reactive oxygen species of interleukin-1 beta, nitric oxide and prostaglandin E(2) production by human chondrocytes. Osteoarthritis Cartilage. 2002 Jul;10(7):547-555.

61. Merkus SL, van Drongelen A, Holte KA, et al. The association between shift work and sick leave: a systematic review. Occup Environ Med. 2012 Oct;69(10):701-712.

62. Pandi-Perumal SR, Srinivasan V, Maestroni GJM, Cardinali DP, Poeggeler B, Hardeland R. Melatonin: nature's most versatile biological signal? FEBS J. 2006 Jul;273(13):2813-2838. 